\title{
Periodontal Measurement on Digital Images \\ Reveals Risk of Overestimation in Clinical Periodontal Probing in Periodontitis Patients
}

Hye-Min Chung

Yonsei University College of Dentistry

Jin-Young Park

Yonsei University College of Dentistry

Kyung-A Ko

Yonsei University College of Dentistry

Seong-Ho Choi

Yonsei University College of Dentistry

Jung-Seok Lee ( $\nabla$ cooldds@gmail.com )

Yonsei University College of Dentistry

\section{Research Article}

Keywords: Periodontitis, gingivitis, imaging, diagnosis

Posted Date: June 30th, 2021

DOI: https://doi.org/10.21203/rs.3.rs-654031/v1

License: (c) (i) This work is licensed under a Creative Commons Attribution 4.0 International License. Read Full License 


\section{Abstract}

Aim: To compare the supra-alveolar gingival dimension (GD) and the clinical pocket probing depth (PD) by combining data from an intraoral scanner (IOS) and cone-beam computed tomography (CBCT), and identify the clinical features affecting the clinical PD.

Materials and Methods: 1,071 sites from 11 patients were selected for whom CBCT, IOS images, and periodontal charts were recorded at the same visit. CBCT and IOS data were superimposed. GD was measured on cross-sectional images of the probed sites. The level of agreement and correlation between GD and PD were assessed for the entire population and also within groups (treated vs untreated, bleeding on probing $[B O P]$ vs no BOP, and PDs of $0-3 \mathrm{~mm}$ vs $4-5 \mathrm{~mm}$ vs $\geq 6 \mathrm{~mm}$ ).

Results: The difference between GD and PD was $0.82 \pm 0.69 \mathrm{~mm}$ (mean \pm standard deviation), and they were positively correlated $(r=0.790, p<0.001)$. The mean difference was smaller for sites with PD $\geq 4 \mathrm{~mm}$ than for those with a PD of $0-3 \mathrm{~mm}$. The correlations between GD and PD were stronger for untreated sites, sites with BOP, and sites with a larger PD.

Conclusion: The pocket PD is prone to overestimation. The digital representation of the supra-alveolar GD is similar to the PD.

\section{Introduction}

Despite many advances in dental technology, the periodontal probe remains the most frequently used diagnostic tool for assessing the health status and attachment level of periodontal tissues [1]. It is well known that the accuracy and reproducibility of the clinical pocket probing depth (PD) can be affected by the probing force, presence of periodontitis, type of periodontal probe, and probing site $[2,3]$. The resulting errors can impact clinical decision-making, especially during longitudinal monitoring of the periodontal status [4].

It is well accepted that the gingival dimension (GD) comprises the 2-mm 'biologic width' of the dentogingival attachment and the 1-mm gingival sulcus [5], whereas the normal probing sulcus depth is considered to range from 1 to $3 \mathrm{~mm}$ in healthy gingiva [6]. Due to the risk of overestimation of clinical probing and the unclear clinical presentation of the cementoenamel junction, clinical data are subject to overestimation of the clinical attachment loss and the severity of periodontal disease. Therefore, in order to appropriately interpret clinical probing data, they need to be clinically compared to the GD.

An intraoral scanner (IOS) is a device that directly captures images of the oral cavity. Numerous studies have shown that an IOS can be highly accurate [7-9]. Also in the field of clinical periodontology, our research team has recently conducted a mucogingival study comparing conventional probe data with digital data obtained using an IOS, and concluded that IOS data are more accurate and reproducible than using a periodontal probe to measure the width of keratinized gingiva [10]. Such an accurate 
representation of the gingival contours can be superimposed with cone-beam computed tomography (CBCT) data to determine the relationship between the gingiva and bone.

Based on the above-described situation, we aimed to compare the PD and the supra-alveolar GD by combining IOS and CBCT data, and to determine the clinical features that affect clinical probing.

\section{Results}

\subsection{Selected samples}

This study analyzed 1,071 sites from 179 teeth of 11 patients. For the group analyses, there were 300 untreated sites and 771 treated sites; 415 sites with BOP and 656 sites with no BOP; and 892 sites with a PD of $0-3 \mathrm{~mm}, 138$ sites with a PD of $4-5 \mathrm{~mm}$, and 41 sites with a PD of $\geq 6 \mathrm{~mm}$ (Table 1 ).

Table 1

Results showing the number of sites in each group, Pearson's correlation coefficients and the mean differences between GD and PD.

\begin{tabular}{|c|c|c|c|c|c|}
\hline & $\begin{array}{l}\text { Sites } \\
\text { (n) }\end{array}$ & $r$ & $p$-value & $\begin{array}{l}\text { Mean } \\
\pm S D\end{array}$ & $p$-value \\
\hline \multicolumn{6}{|c|}{ Periodontal treatment } \\
\hline Treated & 772 & 0.75 & $P<0.001$ & $0.83 \pm 0.68$ & \multirow[t]{2}{*}{$p=0.091$} \\
\hline Non-treated & 299 & 0.86 & $P<0.001$ & $0.76 \pm 0.59$ & \\
\hline \multicolumn{6}{|c|}{ Bleeding on probing site } \\
\hline$(-)$ & 415 & 0.69 & $P<0.001$ & $0.84 \pm 0.66$ & \multirow[t]{2}{*}{$p=0.072$} \\
\hline$(+)$ & 656 & 0.85 & $P<0.001$ & $0.76 \pm 0.64$ & \\
\hline \multicolumn{6}{|c|}{ Clinical probing depth } \\
\hline $0-3 \mathrm{~mm}$ & 892 & 0.42 & $P<0.001$ & $0.87 \pm 0.66^{a}$ & \multirow[t]{3}{*}{$p<0.001$} \\
\hline $4-5 \mathrm{~mm}$ & 138 & 0.66 & $P<0.001$ & $0.51 \pm 0.53^{b}$ & \\
\hline$\geq 6 \mathrm{~mm}$ & 41 & 0.79 & $P<0.001$ & $0.47 \pm 0.52^{b}$ & \\
\hline Total & 1071 & 0.79 & $P<0.001$ & $0.82 \pm 0.69$ & \\
\hline \multicolumn{6}{|c|}{$r=$ Pearson's correlation coefficient } \\
\hline
\end{tabular}

\subsection{Agreement and correlation between GD and PD}


In the Bland-Altman plot (Fig. 2), the difference between GD and PD was $0.82 \pm 0.69 \mathrm{~mm}$ (mean \pm standard deviation). In that figure it can be seen that the coordinates never cross below $0 \mathrm{~mm}$, because GD is always larger than or equal to PD. The limits of agreement were defined as the mean difference plus and minus $1.96 \times$ the standard deviation. Since the lower limit is $0 \mathrm{~mm}$, the two measurement methods had limits of agreement between 2.17 and $0 \mathrm{~mm}$, which can be interpreted as indicating that the difference between GD and PD ranged between 0 and $2.17 \mathrm{~mm}$. This is a highly probable range, since the biologic width is approximately $2 \mathrm{~mm}$, and in the presence of inflammation the difference will tend toward $0 \mathrm{~mm}$. There was a significant positive Pearson's coefficient for the correlation between GD and $\mathrm{PD}(r=0.790, p<0.001)$.

\subsubsection{Treated versus untreated sites (Fig. 3A1 \& A2)}

There was not a statistically significant difference between untreated patients $(0.76 \pm 0.59 \mathrm{~mm})$ and the treated patients $(0.83 \pm 0.68 \mathrm{~mm})$ in terms of the difference between GD and PD. The untreated patient group $(r=0.86, p<0.001)$ showed a significantly stronger correlation than the treated group $(r=0.75, p<$ $0.001)$.

\subsubsection{BOP versus no-BOP sites (Fig. 3B1 \& B2)}

There was not a statistically significant difference between the sites with BOP $(0.76 \pm 0.69 \mathrm{~mm})$ and those without BOP $(0.80 \pm 0.66 \mathrm{~mm})$ in terms of the difference between GD and PD. The correlation was significantly stronger at sites with BOP $(r=0.852, p<0.001)$ than at those without BOP $(r=0.691, p<$ 0.001).

\subsubsection{PDs of $0-3 \mathrm{~mm}$ versus $4-5 \mathrm{~mm}$ versus $\geq 6 \mathrm{~mm}$ (Fig. 3C1, C2, \& C3)}

The difference between GD and PD was largest $(0.87 \pm 0.66 \mathrm{~mm})$ for a PD of $0-3 \mathrm{~mm}$ and smallest for a $P D$ of $\geq 6 \mathrm{~mm}(0.47 \pm 0.52 \mathrm{~mm})$. There were significant differences between the 0 - to $3-\mathrm{mm}$ group and the other groups ( $p<0.001)$, but not between the 4 - to 5 -mm group and the $\geq 6$-mm group $(p=1.00)$. Pearson's coefficients showed that the correlation between GD and PD became stronger as PD increased $(r=0.42,0.66$, and 0.79 for the $0-3,4-5$, and $\geq 6$ mm groups, respectively; $p<0.001)$.

\section{Discussion}

The current study is the first to compare digitized GD and PD values using a large clinical data set. The biologic width can be deduced by subtracting PD from GD. The main findings of this study were (i) the mean difference between GD and PD was smaller than $2 \mathrm{~mm}$, (ii) the difference became smaller at deeper periodontal pockets and at pathologic sites, and (iii) there was a relatively strong positive correlation between the two methods.

In this study, the mean difference between GD and PD was $0.82 \mathrm{~mm}$, which is smaller than the widely accepted biologic width of around $2 \mathrm{~mm}$ as found by Gargiulo et al. in a histologic study of cadavers [5]. 
Although there will be some intra- and interindividual variability in measurements of the biologic width, the value obtained in this study is smaller than that found in a meta-analysis $(2.15-2.30 \mathrm{~mm})$ [11]. One obvious possible explanation is the current study involving patients diagnosed with severe generalized periodontitis. In the presence of inflammation, the probe tip will penetrate the junctional epithelium and stop at the most-coronal part of the noninflamed connective-tissue ligament [12-14]. However, similar results were obtained even at healthy sites with no BOP and a PD of $0-3 \mathrm{~mm}$. Other clinical studies using transgingival probing have also found similarly small biologic widths $[6,11]$. The most likely explanation for this discrepancy would be the rounding error when using a periodontal probe: PDs are often rounded off by the examiner to the nearest millimeter mark on the periodontal probe, thereby introducing subjective bias. Previous studies of the pocket PD and keratinized gingiva width measurements using a periodontal probe have shown that probing values were often overestimated by $1 \mathrm{~mm}[6,10]$. The results of the present study also suggest that examiner bias in pocket probing leads to overestimation.

A Bland-Altman plot includes $95 \%$ limits of agreement to facilitate visual judgements about how well two methods of measurement agree [15]. A smaller range between these two limits indicates better agreement. The limits of agreement in the present study ranged from 0 to $2.17 \mathrm{~mm}$. This is a reasonable range considering that the upper limit coincides with the biologic width. The difference between GD and PD will be closer to the upper limit when the periodontal health is good, whereas it will be nearer to $0 \mathrm{~mm}$ in the presence of inflammation. This was shown clearly by the group analyses performed in this study, in which the correlation between GD and PD was stronger at untreated sites, in the presence of BOP, and when PD was larger than $4 \mathrm{~mm}$. These findings indicate that there was a strong overall correlation between GD and PD. Based on the limits of agreement together with the mean difference proposed by the current study, a digital technique using CBCT and IOS data can be a reliable method for predicting the pocket $\mathrm{PD}$ and for monitoring periodontal health.

One of the limitations of this study was the lack of a gold standard, which would be the histologic measurement, and so future studies are needed to clarify the measurement accuracies of CBCT and IOS data. CBCT-based linear measurements of oral structures have previously been confirmed as being highly accurate and reproducible, with a mean measurement error of less than $0.01 \mathrm{~mm}$ [16]. A study of the accuracy of the same IOS used in the current study revealed its high trueness and precision, with very low errors within the range of $0.01-0.03 \mathrm{~mm}$ [7]. It can therefore be assumed that the present digital measurements were of very high accuracy, and so it is safe to draw conclusions based on the results of this study.

Despite the accuracy of the two digital methods, they have rarely been applied in the periodontal field for diagnostic purposes due to the associated radiation exposure and the low cost-effectiveness of the procedure given that probing is merely a screening tool for disease detection [17]. Nonetheless, digital techniques are being incorporated increasingly frequently in both research and practice for various treatments [18]. It is useful for clinicians to be aware of the relationship between the digitally measured GD and the clinical PD, so that digital measurements can be utilized in the absence of probing data. The 
data obtained in the present study can be useful for medically compromised patients in whom probing may be contraindicated $[19,20]$.

In summary, the clinical pocket PD is prone to overestimation. The supra-alveolar GD measured using digital methods produced similar readings to conventional probing at sites with inflammation and also at healthy sites.

\section{Methods}

\subsection{Study design}

This study retrospectively analyzed clinical data from patients who visited Yonsei University Dental Hospital for the treatment of periodontal disease from 2018 to 2020. All of the included patients had generalized $(>30 \%$ of teeth involved) severe periodontal inflammation as well as $>50 \%$ of alveolar bone loss along with the dental root in radiography, with clinical features of a PD of $>7 \mathrm{~mm}$, clinical attachment loss of $>5 \mathrm{~mm}$, and easy bleeding on probing (BOP). Based on the consensus report of the 2017 World Workshop on the periodontal classification, those patients were diagnosed as generalized periodontitis of stages III and IV with grade C (rapid progression). This study was approved by the Yonsei University Dental Hospital, Institutional Review Board (2-2020-0086), and was conducted in accordance with the Helsinki Declaration. The Institutional Review Board of Yonsei University Dental Hospital abides with Good Clinical Practice and the regulatory requirements, and it has been approved that informed consent can be waivered for this retrospective study.

\subsection{Sample}

The study sample consisted of 11 patients who fulfilled the following eligibility criteria:

1. Diagnosed with or who had received treatment for generalized severe periodontitis.

2. Availability of all three clinical data sets of CBCT, full mouth IOS images, and periodontal charts recorded at the same visit.

3. Full periodontal probing charts including for BOP performed at six sites per tooth.

\subsection{Data acquisition}

All clinical PDs were measured using a color-coded periodontal probe (UNC-15). Probe tips were inserted parallel to the long axis of the tooth with a probing force of $25 \mathrm{~N}$. BOP was considered to be present if bleeding from the probed site was detected shortly after probing. An oral scan was performed (Trios 3 , 3Shape, Copenhagen, Denmark) on the same day as the periodontal probing. CBCT was also performed with the purpose of diagnostic imaging for dental implant therapy at an edentulous site. The CBCT settings were as follows: tube voltage, $80 \mathrm{kVp}$; tube current, $8 \mathrm{~mA}$; exposure time, $17 \mathrm{~s}$; field of view, 102 $\mathrm{mm} \times 102 \mathrm{~mm}$; and voxel size, $0.2 \mathrm{~mm}$. CBCT and IOS data were imported into OnDemand3D software (Cybermed, Seoul, South Korea) and superimposed based on the three sharpest points on the CBCT and 
IOS images. The supra-alveolar GD was defined as the distance between the most-coronal point of the marginal gingiva and the underlying alveolar bone in the cross-sectional image obtained in the apicocoronal axis of the tooth (Fig. 1). Cross-sectional images were obtained the following six sites of each tooth representing the probed sites: mesiobuccal, midbuccal, distobuccal, mesiolingual, midlingual, and distolingual. All measurements were made by the same trained examiner, and were repeated twice with a 2-week interval (intraexaminer correlation coefficient $=0.89$, confidence interval $=0.88-0.91$ ).

\subsection{Outcome}

The main outcome of the study was the level of agreement between GD and PD as quantified by the mean difference between the two methods. The coefficient for the correlation between GD and PD was also calculated to evaluate the strength of the association. The sites were analyzed for the entire population and then divided into groups for evaluations according to the following three clinical features as potential modifying factors:

i. Patients treated $(n=7)$ versus patients not treated $(n=4)$ for periodontitis.

ii. Sites with BOP versus sites without BOP.

iii. Sites with PDs of $0-3 \mathrm{~mm}$ versus $4-5 \mathrm{~mm}$ versus $\geq 6 \mathrm{~mm}$.

\subsection{Statistical analysis}

The Bland-Altman method was used to analyze the degree of agreement between GD and PD. Pearson's correlation coefficient ( $r$ ) was used to analyze the correlation between the GD and PD. Mean differences between two groups were compared using the unpaired $t$-test, while those between three groups were compared using one-way ANOVA and the Bonferroni correction. Values of $r$ were statistically compared between groups with a 95\% confidence level using the SPSS program (IBM SPSS Statistics for Windows, version 22.0, IBM Corporation, Armonk, NY).

\section{Declarations}

Acknowledgements

This study was supported by National Research Foundation of Korea (NRF) funded by the Ministry of Science, ICT \& Future Planning (NRF-2019R1A2C4069942).

Conflict of interest

Authors declare that there was no conflict of interest in regards to this study.

\section{References}

1. Al Shayeb, K.N., W. Turner, and D.G. Gillam, Periodontal probing: a review. Prim Dent J, 2014. 3(3): p. 25-9. 
2. Gupta, N., S.K. Rath, and P. Lohra, Comparative evaluation of accuracy of periodontal probing depth and attachment levels using a Florida probe versus traditional probes. Med J Armed Forces India, 2015. 71(4): p. 352-8.

3. Erriu, M., et al., Probing depth in periodontal pockets: In vitro evaluation of contributions to variability due to probe type and operator skill. Proc Inst Mech Eng H, 2015. 229(10): p. 743-9.

4. Greenstein, G., Contemporary interpretation of probing depth assessments: diagnostic and therapeutic implications. A literature review. J Periodontol, 1997. 68(12): p. 1194-205.

5. Gargiulo, A.W., F.M. Wentz, and B. Orban, Dimensions and Relations of the Dentogingival Junction in Humans. The Journal of Periodontology, 1961. 32(3): p. 261-267.

6. Hamasni, F.M. and F. El Hajj, Comparison of the Clinical Biological Width with the Published Standard Histologic Mean Values. J Int Soc Prev Community Dent, 2017. 7(5): p. 264-271.

7. Winkler, J. and N. Gkantidis, Trueness and precision of intraoral scanners in the maxillary dental arch: an in vivo analysis. Sci Rep, 2020. 10(1): p. 1172.

8. Treesh, J.C., et al., Complete-arch accuracy of intraoral scanners. J Prosthet Dent, 2018. 120(3): p. 382-388.

9. Celeghin, G., et al., Complete-Arch Accuracy of Four Intraoral Scanners: An In Vitro Study. Healthcare (Basel), 2021. 9(3).

10. Lee, J.S., et al., Digital scanning is more accurate than using a periodontal probe to measure the keratinized tissue width. Sci Rep, 2020. 10(1): p. 3665.

11. Schmidt, J.C., et al., Biologic width dimensions-a systematic review. J Clin Periodontol, 2013. 40(5): p. 493-504.

12. Caton, J., G. Greenstein, and A.M. Polson, Depth of periodontal probe penetration related to clinical and histologic signs of gingival inflammation. J Periodontol, 1981. 52(10): p. 626-9.

13. Magnusson, I. and M.A. Listgarten, Histological evaluation of probing depth following periodontal treatment. J Clin Periodontol, 1980. 7(1): p. 26-31.

14. Listgarten, M.A., R. Mao, and P.J. Robinson, Periodontal probing and the relationship of the probe tip to periodontal tissues. J Periodontol, 1976. 47(9): p. 511-3.

15. Myles, P.S. and J. Cui, Using the Bland-Altman method to measure agreement with repeated measures. Br J Anaesth, 2007. 99(3): p. 309-11.

16. Garcia-Sanz, V., et al., Accuracy and Reliability of Cone-Beam Computed Tomography for Linear and Volumetric Mandibular Condyle Measurements. A Human Cadaver Study. Sci Rep, 2017. 7(1): p. 
17. Greenstein, G., Current interpretations of periodontal probing evaluations: diagnostic and therapeutic implications. Compend Contin Educ Dent, 2005. 26(6): p. 381-2, 384, 387-90 passim; quiz 398-9.

18. Spagnuolo, G. and R. Sorrentino, The Role of Digital Devices in Dentistry: Clinical Trends and Scientific Evidences. J Clin Med, 2020. 9(6).

19. Royzman, D., et al., The effect of aspirin intake on bleeding on probing in patients with gingivitis. J Periodontol, 2004. 75(5): p. 679-84.

20. Daly, C.G., et al., Bacteremia due to periodontal probing: a clinical and microbiological investigation. J Periodontol, 2001. 72(2): p. 210-4.

\section{Figures}
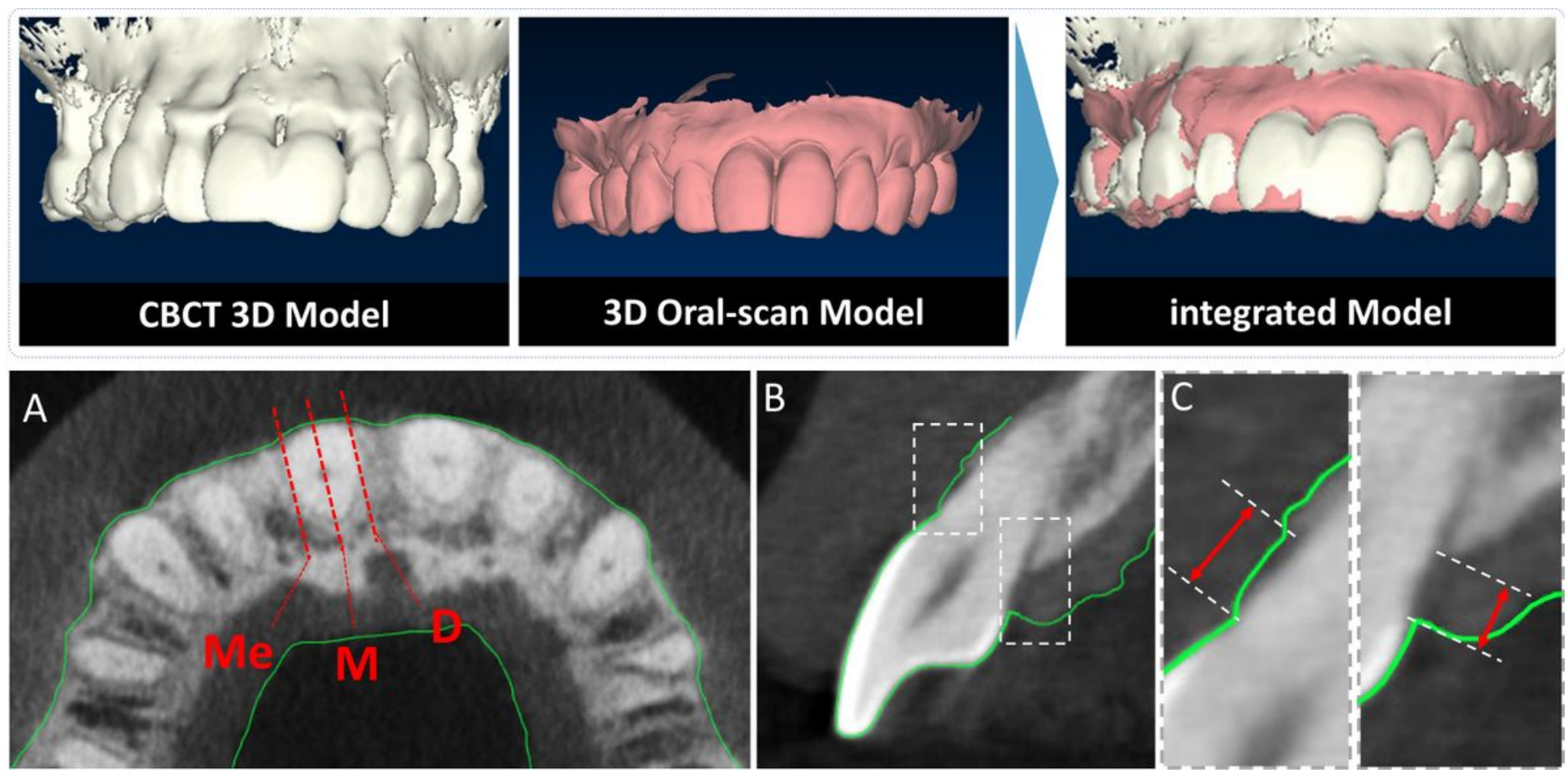

Figure 1

Cone-beam computed tomography (CBCT) images were acquired and integrated with an oral-scan model using OnDemand3D software. The supra-alveolar gingival dimension (GD) was measured. (A) Axial image showing the positions selected for vertical cross-sectioning (mesial, middle, and distal). (B) Crosssectional image of the dentogingival unit for GD measurement. (C) GD was defined as the distance between the most-coronal point of the marginal gingiva and the underlying alveolar bone. 
6

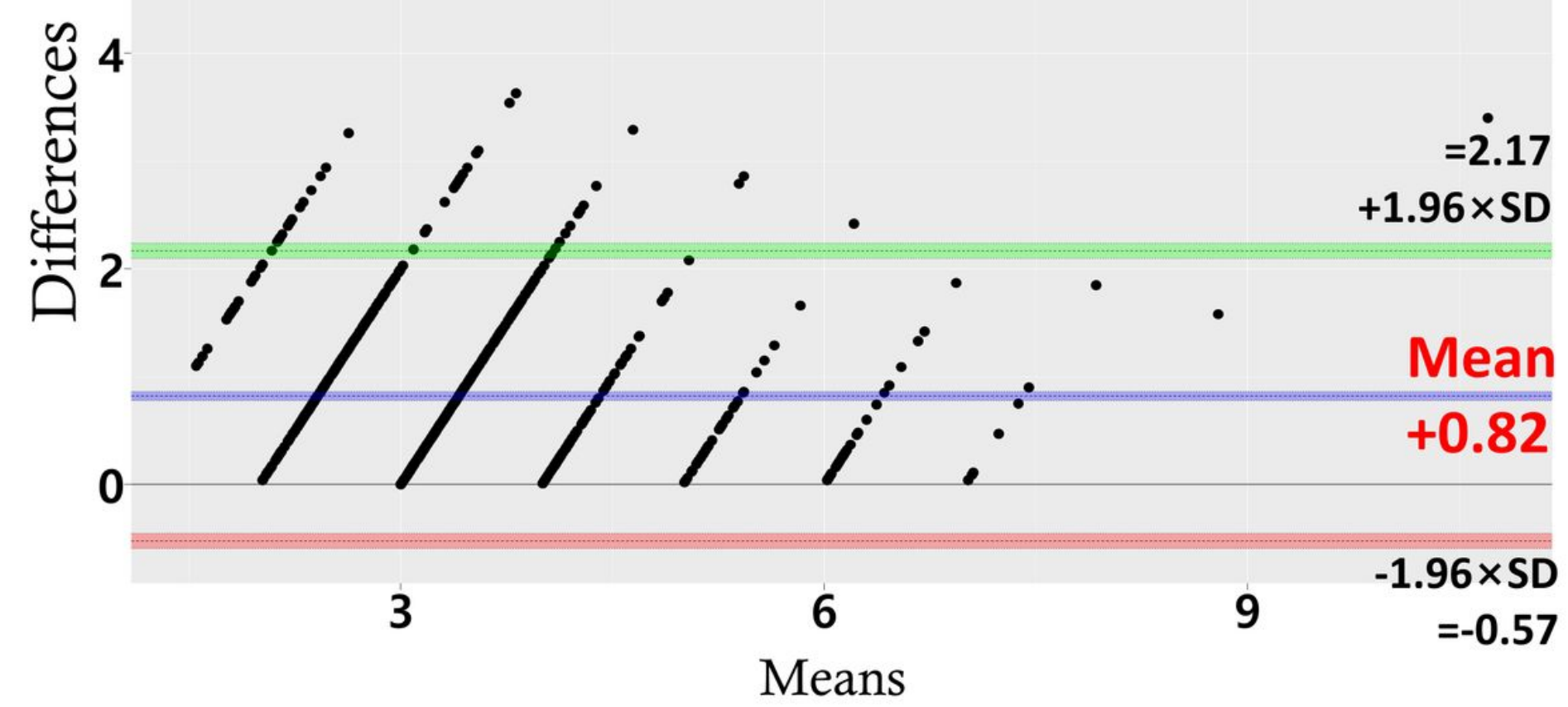

Figure 2

Bland-Altman plot of the mean of GD and probing depth (PD) (x-axis) versus the difference between GD and PD (y-axis). The mean difference (blue line) between GD and PD was $0.82 \mathrm{~mm}$. The limits of agreement (green and red lines) are defined as the mean difference plus and minus $1.96 \times$ the standard deviation. The coordinates never cross below $0 \mathrm{~mm}$, because GD is always larger than or equal to PD. Since the lower limit is $0 \mathrm{~mm}$, the two measurement methods had limits of agreement between 2.17 and $0 \mathrm{~mm}$. 

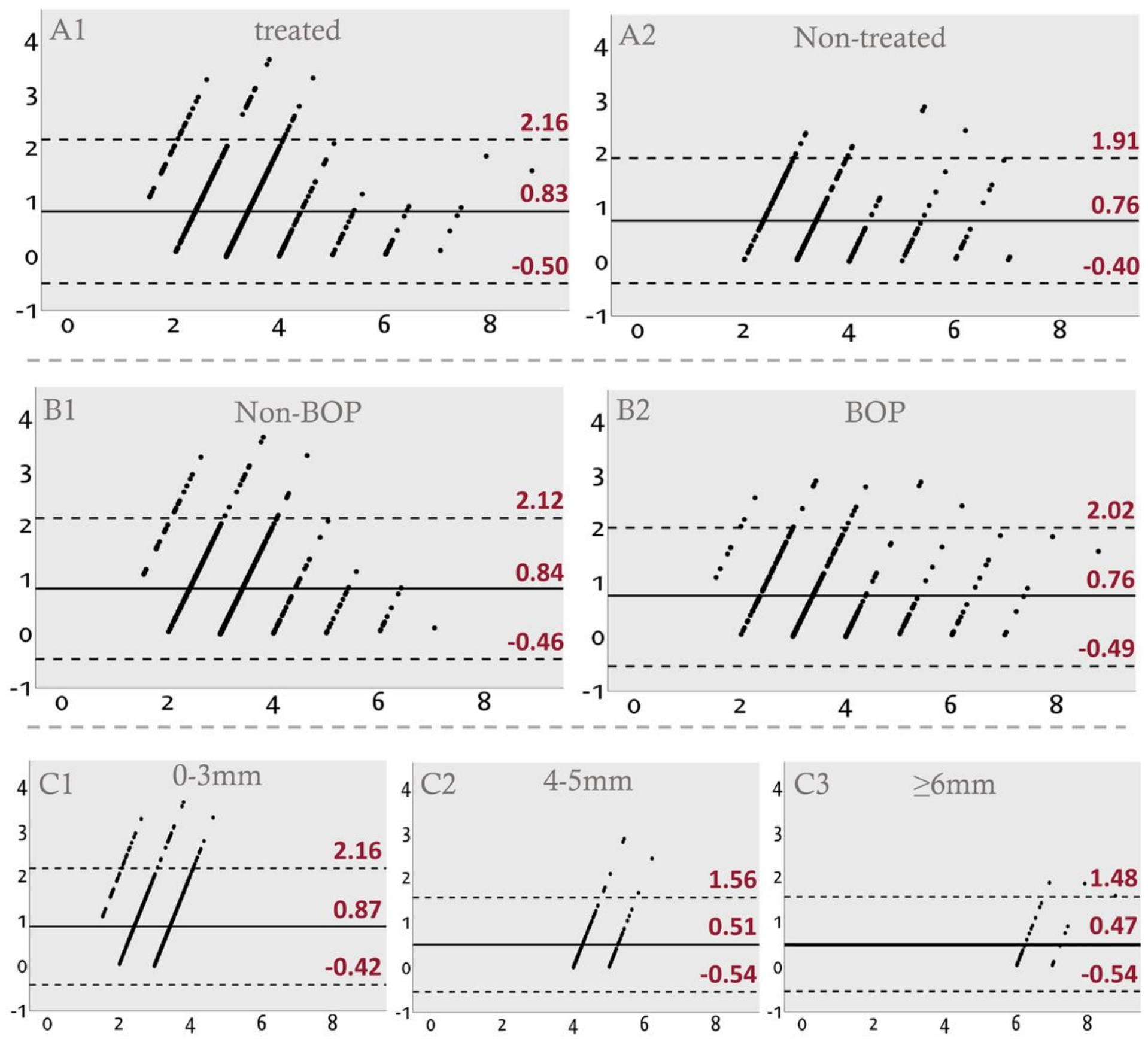

Figure 3

Bland-Altman plots for each group according to clinical features affecting the clinical PD. (A1) Sites treated for periodontitis. (A2) Sites with periodontitis that were not treated. (B1) Sites without BOP. (B2) Sites with BOP. (C1) Sites with a PD of 0-3 mm. (C2) Sites with a PD of 4-5 mm. (C3) Sites with a PD of $\geq 6 \mathrm{~mm}$. 